\title{
Chitosan and Quat-188 Modified Chitosan Poly[acrylic acidd] Semi-Interpenetrating Network For Controlled Release of Drugs
}

\author{
Christian Gonzales $^{1}$, Mari Kaira O. Leal ${ }^{1}$, Ivy Marie C. Andalis ${ }^{1}$, Modesto T. Chua ${ }^{1}$, \\ Takahiko Nakaoki ${ }^{2}$, and Soma Chakraborty ${ }^{*}$ \\ ${ }^{1}$ Department of Chemistry, School of Science and Engineering, Ateneo de Manila University, Loyola Heights, \\ Quezon City, Philippines \\ ${ }^{2}$ Department of Materials Chemistry, Ryukoku University, Seta, Otsu, Japan
}

\begin{abstract}
Semi-interpenetrating polymer networks (semi-IPNs) composed of polyacrylic acid (PAA), and chitosan or N-(3-chloro-2-hydroxypropyl)trimethylammonium chloride (Quat-188) modified chitosan were synthesized. To fabricate the semi-IPN, acrylic acid (AA) was polymerized and crosslinked in the presence of unmodified and Quat-188 modified chitosan. The wet strength of the semi-IPNs improved with the increase in molecular weight of chitosan, chitosan to PAA ratio and by Quat-188 modification. Both modified and unmodified semi-IPNs swelled in buffer solutions. Swelling was $\mathrm{pH}$ dependent. The mode of encapsulation and release of two different types of drugs from these semi IPNs was studied. Effects of various parameters on encapsulation and release of $\mathrm{AgNO}_{3}$ and mafenide acetate from these semi-IPNs were investigated. The semi-IPN hydrogel encapsulated $10 \mathrm{mmol} / \mathrm{L} \mathrm{AgNO}_{3}(100 \%$ of added drug) in $5 \mathrm{~min}$ and released $4 \%$ of it in $2 \mathrm{~h}$. In case of mafenide acetate, $\mathrm{pH}$ dependent encapsulation and controlled release was observed. It was observed that drug-semi IPN interaction strongly influences the encapsulation and release behavior. Freezable water associated with the hydrogels also played an important role for the encapsulation and release of drug.
\end{abstract}

Keywords: chitosan; controlled drug delivery; hydrogel; Quat-188; poly(acrylic acid)

\section{INTRODUCTION}

Chitosan, a derivative of chitin, due to its availability, biodegradability, and biocompatibility, has been utilized for numerous pharmaceutical and biomedical applications that includes controlled drug delivery (Park et al. 2010; Quiñones et al. 2011; Li et al. 2011), scaffolding (Li et al. 2010; Wu et al. 2011; Jaykumar et al. 2011) and as sensor (Odaci et al., 2008; Njagi et al. 2010).

Some of the above mentioned applications use chitosan in semi-IPN form. Semi-IPN hydrogels are formed by the combination of two polymers. One forms a network due to crosslinking, the other polymer in linear form penetrates into the network of the first one. 
These polymers influence the properties of each other. Formation of semi-IPN and IPNs of chitosan with various synthetic polymers poly(methacryloylglycylglycine)(Dash et al., 2012), poly(N-isopropylacrylamide) (Guo et al., 2007; Henares et al., 2010) and polyacrylamide(Dragan et al., 2013) has been reported.

Association of polyacrylic acid (PAA) with chitosan improves the swelling behavior and mucoadhesiveness of chitosan, hence chitosan in combination with PAA in the form of polyelectrolyte complex has been studied for various applications( Sailaja et al. 2006; Ahn et al. 2002; Hu et al. 2007).

N-(3-chloro-2-hydroxypropyl)trimethylammonium chloride (Quat-188) is a quaternary ammonium chloride that is used to modify polymers to produce quaternary ammonium side-groups. It is commonly used in cosmetics, the paper industry, the textile industry, and water management industries as a coagulant and an antistatic agent. Quaternary ammonium derivative of chitosan is used for various industrial and pharmaceutical applications (Jia and Wu, 2006). Quaternization helps chitosan to retain positive charge permanently and improves its interaction with water. Improvement of antimicrobial property of chitosan upon modified using Quat-188 has been reported by Qin et al.(Qin et al. 2004). In alkaline medium, Quat-188 is converted to glycidyltrimethylammonium chloride (GTMAC) which then reacts with chitosan.

Our project aimed to fabricate of chitosan and chitosan $\mathrm{N}$-(2-hydroxypropyl)trimethylammonium chloride (modified chitosan) PAA semi IPN that can be and to study the impact of swelling, drug-polymer interaction and the size of the drug on the encapsulation and release behavior from Chitosan PAA matrix. $\mathrm{AgNO}_{3}$ and mafenide acetate were used as the model drugs. The effect of freezable and nonfreezable water on drug-polymer interaction has also been investigated using cryo DSC. Hydrogels reported in this study is intended for application as transdermal drug delivery patch.

\section{EXPERIMENTAL}

Chemicals. Medium and high molecular weight chitosan, with viscosity of 200-800 centipoise (cp) and 800-2000cp respectively and $75-85 \%$ deacylated, acrylic acid, N,Nmethylenebisacrylamide and mafenide acetate were purchased from Sigma Aldrich. $\mathrm{AgNO}_{3}$ was purchased from J.T. Baker. Quat-188 was obtained from Rohm and Haas Company. All chemicals were used without prior purification.

Instruments. $1 \mathrm{H}$ NMR Spectroscopy. ${ }^{1} \mathrm{H}$ NMR spectra were recorded at $25^{\circ} \mathrm{C}$ on a JEOL Lambda $400 \mathrm{MHz} .{ }^{1} \mathrm{H}$ NMR chemical shifts in parts per million (ppm) were referenced relative to tetramethylsilane (TMS). $8.0 \mathrm{wt} \%$ of each sample was dissolved in deuterated water $\left(\mathrm{D}_{2} \mathrm{O}\right)$. A trace amount of acetic acid was added to the sample to make chitosan soluble in $\mathrm{D}_{2} \mathrm{O}$.

Cryo Differential Scanning Calorimetry (Cryo DSC). Rigaku DSC 8230D was used to perform cryo DSC. The measurements were carried out from $100{ }^{\circ} \mathrm{C}$ to $20^{\circ} \mathrm{C}$ at a cooling rate of 10 ${ }^{\circ} \mathrm{C} / \mathrm{min}$ under a nitrogen flow. Gel was closely sealed in an aluminum pan to prevent the evaporation of water.

UV-Vis Spectroscopy. The amount of mafenide acetate loaded and release by the particles was quantified using UV-Vis analysis with a Shimadzu 2401 PC UV Spectrophotometer at $222 \mathrm{~nm}$ using calibration curve method.

A calibration curve was first prepared using 1 , $2,4,5,6,8,10$, and $15 \mathrm{ppm}$ of mafenide acetate solution in $\mathrm{pH} 7$ and 5 buffer. Then, the amount of drug loaded into the hydrogel was calculated by subtracting the amount of drug left in the supernatant solution determined via UV-Vis absorbance at $222 \mathrm{~nm}$ from the initial amount of drug added. Supernatant solution was diluted 3 times before subjecting the solution to UV-vis analysis. The amount of loaded drug was quantified as the percentage ratio of the mass of drug loaded to the mass of the initial drug concentration. The amount of mafenide acetate released was also quantified from the 
UV-Vis spectroscopy of the supernatant. Percent of drug released was calculated as the ratio of amount (mass) of drug in supernatant versus the amount (mass) of loaded drug in the hydrogels.

Inductively Coupled Plasma (ICP) Spectrometry. Inductively coupled plasma spectrometry was used to study the loading and release of $\mathrm{AgNO}_{3}$. The supernatant liquid from the drug loading and release experiments were subjected to ICP-spectrometry using Shimadzu ICPS 7510 instrument. Supernatant liquid sample $(2 \mathrm{~mL})$ was digested with 0.02 $\mathrm{mL}$ concentrated $\mathrm{HNO}_{3}$ followed by adjustment with $5 \mathrm{~mL}$ distilled water. Argon was used as the carrier gas with the flow rate of $0.7 \mathrm{~L} / \mathrm{min}$. It was also used as the cooling gas with the flow rate of $1.4 \mathrm{~L} / \mathrm{min}$. Silver ion was analyzed at $328.068 \mathrm{~nm}$. Amount of silver ion was quantified using calibration curve method.

Swelling, drug loading and drug release experiments were performed in duplicate and the mean values are reported.

Methodology. Modification of Chitosan with Quat-188. To prepare Quat-188 modified chitosan, $3.75 \mathrm{mmol}$ per unit chitosan was placed in a round bottom flask. To it 1.88 mmol of Quat-188 was added along with $10 \mathrm{~mL}$ of deionized water. The $\mathrm{pH}$ of the mixture was adjusted to $\mathrm{pH} 9$ by dropwise addition of $0.5 \mathrm{M} \mathrm{NaOH}$. The flask was sealed, purged with nitrogen and the reaction was performed at $70^{\circ} \mathrm{C}$ for $48 \mathrm{~h}$ under constant stirring. After the reaction, the system was neutralized to $\mathrm{pH} 7$, centrifuged and excess water was removed by decantation. Quat-188 modified chitosan was further freeze dried using Labconco Freeze Dry System/Freezone 45 freeze drier. The dried product was characterized using ${ }^{1} \mathrm{H}$ NMR spectroscopy. In past synthesis of Quat-188 derivative of chitosan has been reported using iodine (Sajomsang et al. 2009). 1M pH 9 buffer was prepared by mixing 0.477 moles of monosodium phosphate and 0.523 moles of disodium phosphate in $1 \mathrm{~L}$ of water.

Synthesis of Chitosan-Polyacrylic Acid Semi-IPN. To synthesize the semi-IPN with 2:1 molar ratio of chitosan to PAA and $5 \mathrm{~mol} \%$ crosslinking, $0.146 \mathrm{mmol}$ of acrylic acid (AA), $0.1400 \mathrm{~g}$ of chitosan and $5 \mathrm{~mL}$ of deionized water were transferred in a round-bottomed flask. The flask was sealed using a rubber septum and purged with $\mathrm{N}_{2}$ gas. The system was stirred at room temperature for $1 \mathrm{~h}$ and then N,N'-methylenebisacrylamide was added to the homogenized system using a syringe. The amount of N,N'-methylenebisacrylamide added was $5 \mathrm{~mol} \%$ with respect to the amount of AA. Polymerization was initiated by addition of $4.0 \mathrm{mg}$ of potassium persulfate dissolved in $1 \mathrm{~mL}$ of deionized water. The reactions were performed at $70^{\circ} \mathrm{C}$ for 6 hours. After $6 \mathrm{~h}$ the product was transferred to a small aluminum mold and was oven dried until a constant weight was obtained. The final product was in the form of a very thin film. Above procedure was also adopted for the fabrication of semi-IPN of modified chitosan with PAA where Quat-188-modified chitosan replaced unmodified chitosan.

Swelling Study. The swelling behavior of the synthesized Semi-IPNs was studied by allowing the dry hydrogels $(1 \mathrm{~g})$ to swell in $15 \mathrm{~mL}$ of deionized water $(\mathrm{pH} 5.5)$ and $\mathrm{pH} 7$ phosphate buffer solutions for certain period of time in aluminum mold. The hydrogels were then removed, pat dried and the weight of the swelled hydrogel was determined. Swelling percentage ( $\%$ swelling) was determined using the formula:

$$
\% \text { swelling }=\frac{\text { weight of swelled hydrogel }- \text { weight of dry hydrogel }}{\text { weight of dry hydrogel }} \times 100 \%
$$

Drug-loading study. To load the drug, $2 \mathrm{~mL}$ of buffer solution was added to semi-IPN hydrogels in the mold and equilibrated for
$30 \mathrm{~min}$ on a shaker then the aqueous solution of the drug of desired concentration $(\mathrm{mg} / \mathrm{mL})$ was added to the buffer. The systems were 
further equilibrated on a shaker for certain period of time. The drug-loaded hydrogels were centrifuged at 10,000 rpm to allow them to settle down. The supernatant liquid was decanted and amount of residual drug present in the supernatant was quantified using UVvis spectroscopy and ICP for mafenide acetate and $\mathrm{AgNO}_{3}$ respectively.

Drug release study. Drug loaded semi IPN hydrogels were equilibrated in $2 \mathrm{~mL}$ buffer solution for certain period of time. The hydrogels were then centrifuged at 10,000 rpm for 30 minutes to allow them to settle down. The supernatant liquid was decanted and the amount of drug present in the supernatant was quantified using UV-vis spectroscopy and ICP for mafenide acetate and $\mathrm{AgNO}_{3}$ respectively.

\section{RESULTS AND DISCUSSION}

Synthesis of Quat-188 modified Chitosan. Chitosan was modified by reacting chitosan with Quat-188 in the presence of $\mathrm{NaOH}$ solution at $\mathrm{pH}$ 9. Chitosan was modified with Quat-188 to enhance its cationic nature for efficient interaction with polyanionic polyacrylic acid. After performing the reaction for $48 \mathrm{~h}$, the product was characterized using ${ }^{1} \mathrm{H}$ NMR spectroscopy. Reaction scheme is shown in Figure 1.
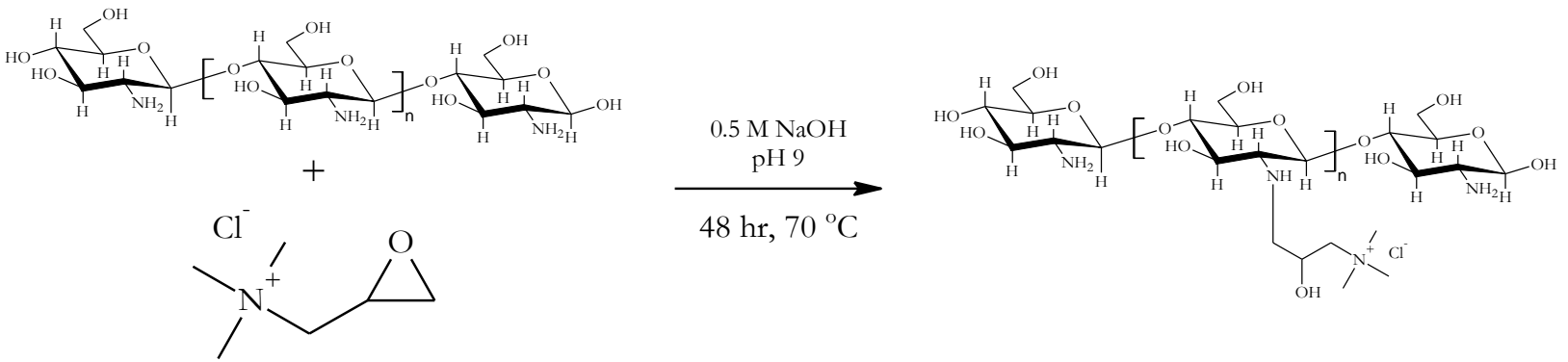

Figure 1. Reaction between Chitosan and Quat-188 to form Quat-188 modified Chitosan.

Figure 2 shows the ${ }^{1} \mathrm{H}$ NMR spectrum of Quat-188 modified chitosan in $\mathrm{D}_{2} \mathrm{O} .{ }^{1} \mathrm{H}$ NMR spectral data of the product are as follows: ${ }^{1} \mathrm{H}$ NMR in $\mathrm{D}_{2} \mathrm{O}$ (in ppm): 4.3-4.25 [a: $\mathrm{C} \underline{\mathrm{H}}-\mathrm{O}-$ of chitosan], 2.7-2.85 [b: $\mathrm{C} \underline{\mathrm{H}}-\mathrm{NH}_{2}$ of chitosan], 3.6-4.25 [c : $\mathrm{C} \underline{\mathrm{H}}-\mathrm{OH}$; d: $-\mathrm{O}-\mathrm{C} \underline{\mathrm{H}}$; e: $\mathrm{C} \underline{\mathrm{H}}-$ $\mathrm{CH}_{2} \mathrm{OH} ; \mathrm{f}: \mathrm{CH}_{2} \mathrm{OH}$ of chitosan]: , 3.4-3.5[1:$\mathrm{NH}-\underline{\mathrm{C}}_{2}-$ of Quat-188], 4.3-4.25[2: $\mathrm{C} \underline{\mathrm{H}}-\mathrm{OH}$ of Quat-188], 3.55-3.6[3: $\mathrm{C}_{2}-\mathrm{N}^{+}\left(\mathrm{CH}_{3}\right)$ of Quat-188],3.0-3.3[4: $\left(\mathrm{C}_{3}\right)_{3}-\mathrm{N}^{+}$of Quat-188].

The peak position of 1:NH-C $\underline{\mathrm{H}}_{2}$ and 2: $\mathrm{CH}-$ $\mathrm{OH}$ of unreacted Quat-188 are 3.6ppm and $4.67 \mathrm{ppm}$ respectively which showed downfield shift when Quat-188 was reacted with chitosan.

Swelling Behavior of the semi IPNs. Swelling determines the water retention capacity of the hydrogel and also the encapsulation and release efficiency. Hydrogels are required to swell to encapsulate the attributes, however excessive swelling causes the hydrogel to rupture. Hence the swelling behavior of the semi IPNs was
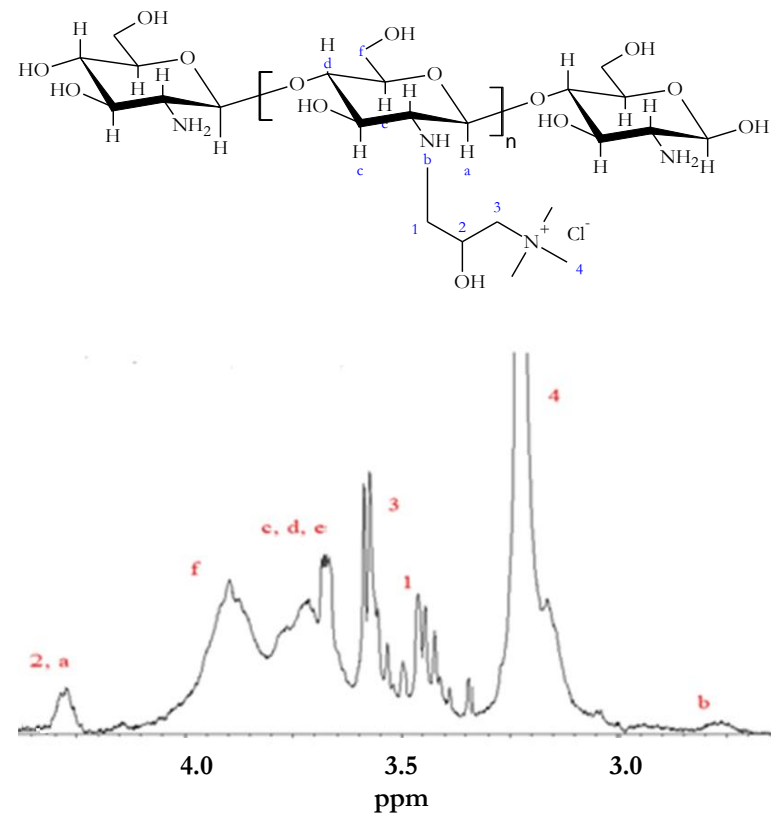

Figure 2. ${ }^{1} H$ NMR spectrum of Quat-188 modified chitosan in $\mathrm{D}_{2} \mathrm{O}$.

studied to ensure that the gels are capable of drug encapsulation without rupturing and the effect of swelling on drug encapsulation and release. It was observed that molecular weight of chitosan, the amount of chitosan and 
presence of Quat-188 had profound influence on the swelling behavior of the hydrogels. As summarized in Table 1, it was observed that when medium molecular weight chitosan was used the hydrogels swelled extensively and ruptured within a short period of time. Rupturing could not be prevented by enhancing the amount of crosslinker. However, increase in the molecular weight of the chitosan and the amount of chitosan increased the wet strength of the hydrogel, thus prevented rupturing of the hydrogels.
Chitosan has the ability to form strong films thus addition of chitosan prevented the rupturing of the hydrogels. It was also noticed that modification of chitosan using Quat-188 also prevented rupturing. It can be hypothesized that when chitosan was modified with Quat-188, it induced more positive charge to chitosan through quaternary ammonium ion and enhanced the interaction between the poly anionic PAA and polycationic chitosan thus improved the wet strength of the hydrogels.

Table 1. Swelling behavior of Chitosan PAA semi IPNs.

\begin{tabular}{ccccc}
\hline Entry & $\begin{array}{c}\text { Chitosan: AA } \\
(\mathbf{m o l} / \mathbf{m o l})\end{array}$ & $\begin{array}{c}\text { Crosslinker } \\
\text { (mol/mol) } \\
\text { w.r.t. AA }\end{array}$ & $\begin{array}{c}\text { Chitosan mol. } \\
\text { weight }\end{array}$ & $\begin{array}{c}\text { Time taken to } \\
\text { rupture }\end{array}$ \\
\hline 1 & $1: 1$ & $5 \%$ & Medium & 5 min \\
\hline 2 & $1: 1$ & $10 \%$ & Medium & $30 \mathrm{~min}$ \\
\hline 3 & $1: 1$ & $30 \%$ & Medium & $2 \mathrm{~h}$ \\
\hline 4 & $1: 1$ & $30 \%$ & High & No rupture \\
\hline 5 & $2: 1$ & $30 \%$ & Medium & No rupture \\
\hline 6 & $1: 1$ & $30 \%$ & Medium* & No rupture \\
\hline
\end{tabular}

*Quat -188 modified medium molecular weight chitosan was used

The swelling behavior of semi-IPNs with 2:1 mol ratio of chitosan to PAA was studied systematically as function of time. As shown in Figure 3, the swelling was $\mathrm{pH}$ dependent. Chitosan semi-IPN swelled more in $\mathrm{pH} 7$ than in $\mathrm{pH}$ solution. It swelled in acidic $\mathrm{pH}$ due to the repulsion between the ammonium ions of chitosan, in alkaline $\mathrm{pH}$ repulsion between the carboxylate ions of PAA swelled the semiIPNs. Repulsion between the carboxylate ions was predominant over the repulsion between the ammonium ions, thus the hydrogels swelled more in $\mathrm{pH}$ 7. Reverse trend was observed for the Quat-188 modified chitosan semi IPN. Under acidic condition the repulsion between the ammonium ions of chitosan and quaternary ammonium of Quat188 resulted in enhanced swelling. Modified chitosan semi-IPN swelled more than unmodified chitosan semi-IPN due to the presence of quaternary ammonium ion. A drastic decrease in \%swelling was observed for the modified chitosan hydrogel after $1 \mathrm{~h}$ of equilibration.

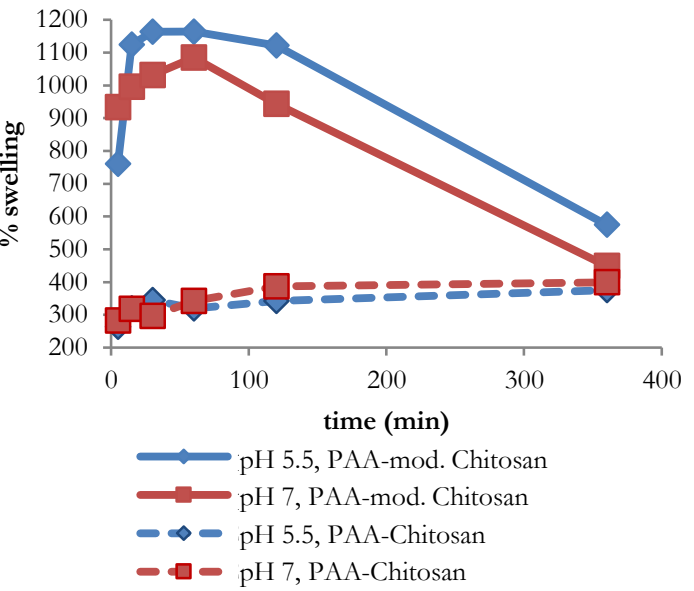

Figure 3. Swelling of chitosan and modified chitosan $P A A$ semi IPNs as function of time and $p H$.

Cryo DSC. In general, water in the hydrogel is classified into two states; freezable and nonfreezable water. It has been established that non-freezable water has a strong interaction with polymer chain (Nakaoki et al 2008; Nakano et al 2011) and encapsulation and release of drugs is dependent on the amount of freezable water in the hydrogel. Therefore cryo DSC measurement was carried out to 
estimate the amount of freezable water and its impact on drug encapsulation and release. Figure 4 shows the DSC thermogram of the melting process of water in the gel. The melting enthalpy of water in the hydrogel comes from the freezable water. The weight content of freezable water $\left(\mathrm{w}_{\mathrm{F}}\right)$ was estimated by the following equation

$$
\mathrm{w}_{\mathrm{F}}=\frac{\Delta H_{\mathrm{obs}}}{\Delta H_{\mathrm{water}}}
$$

where $\Delta \mathrm{H}_{\mathrm{obs}}$ and $\Delta \mathrm{H}_{\text {water }}$ denote the melting enthalpies of observed and pure water, respectively. The weight contents of freezable water for modified and native chitosan were 86 and $81 \%$, respectively. Modified chitosan contains larger amount of freezable water. Therefore it is expected that modified chitosan will allow more efficient encapsulation and release of drugs.

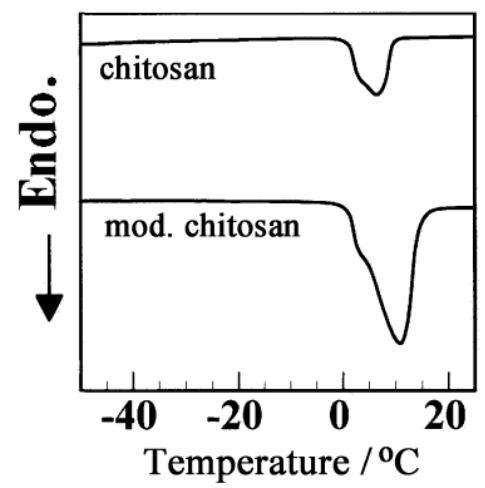

Figure 4. DSC thermogram of the melting process of water in the chitosan and modified chitosan gels.

Drug encapsulation and release. $\mathrm{AgNO}_{3}$ and mafenide acetate were the two prototype drugs that were used for our study. These drugs are used to treat the wound due to injury from burn by topical application. Instead of their direct and repeated application, their controlled release at the site of injury from a hydrogel patch might serve as an efficient way of treating injury due to burn. Chitosan, owing to its strong film forming ability and haemostatic properties has also been explored as a primary component of wound dressing patch (Bochicchio et al. 2009).The group studied the encapsulation and release of these two drugs which differ in size and the nature of interaction with the semi IPN hydrogel of chitosan while both having the burn wound treatment application.

$\mathrm{AgNO}_{3}$ encapsulation and release. A rapid incorporation of $\mathrm{AgNO}_{3}$ inside the modified and unmodified chitosan hydrogels was observed within a short interval of time (Table 2). However, no noticeable release of $\mathrm{AgNO}_{3}$ was observed from the hydrogel with $10 \mathrm{mg}$ of $\mathrm{AgNO}_{3}$ even after $6 \mathrm{~h}$ as shown in Table 3.

Table 2. Encapsulation of $\mathrm{AgNO}_{3}$ by Chitosan PAA semi IPNs.

\begin{tabular}{cccc}
\hline Entry & $\begin{array}{c}\text { Initial } \\
\text { AgNO } \\
(\mathbf{m g})\end{array}$ & $\begin{array}{c}\text { Equilibration } \\
\text { Time } \\
\text { (mins.) }\end{array}$ & $\begin{array}{c}\text { AgNO } \\
\text { incorporated } \\
\text { (mg) } \\
\text { (\% incorporation) }\end{array}$ \\
\hline 1 & 10 & 10 & $10(100 \%)$ \\
\hline 2 & 30 & 10 & $26.7(89 \%)$ \\
\hline 3 & 30 & 120 & $29.1(97 \%)$ \\
\hline 4 & 10 & 10 & $10(100 \%)$ \\
\hline 5 & 30 & 10 & $26.1(87 \%)$ \\
\hline 6 & 30 & 120 & $27(90 \%)$ \\
\hline
\end{tabular}

Table 3. Release of $\mathrm{AgNO}_{3}$ from Chitosan PAA semi IPNs.

\begin{tabular}{cccc}
\hline Entry & pH & $\begin{array}{c}\text { Equilibration } \\
\text { Time } \\
\text { (mins.) }\end{array}$ & $\begin{array}{c}\text { AgNO }_{3} \\
\text { released } \\
(\text { mg) }(\% \text { release) }\end{array}$ \\
\hline & Modified Chitosan PAA hydrogel \\
\hline 1 & 5.5 & 120 & $0.4(4 \%)$ \\
\hline 2 & 5.5 & 360 & $0.4(4 \%)$ \\
\hline 3 & 7 & 120 & $0.16(1.6 \%)$ \\
\hline 3 & Unmodified Chitosan PAA hydrogel \\
\hline 4 & 5.5 & 120 & - \\
\hline
\end{tabular}

Maximum release of $4 \% \mathrm{AgNO}_{3}$ in $2 \mathrm{~h}$ was observed from modified chitosan PAA semiIPN which can be attributed to immense swelling of the network and the presence of 


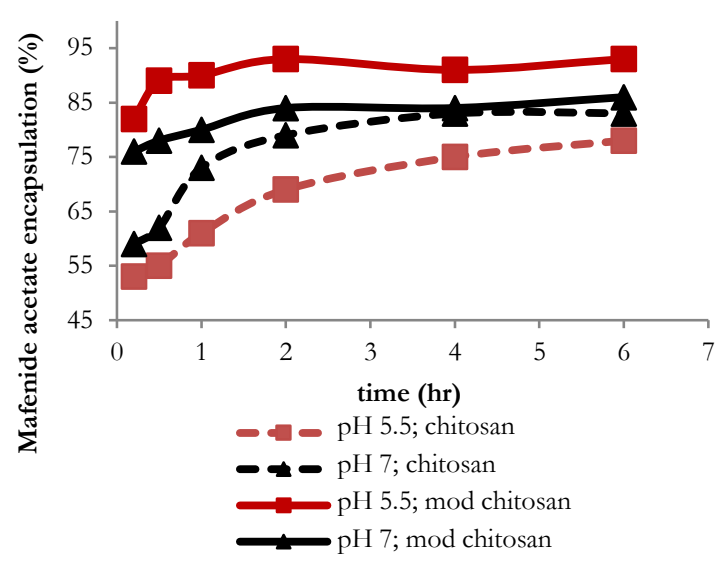

Figure 5. Mafenide acetate encapsulation by chitosan and modified chitosan PAA semi IPNs as function of time and $p H$.

freezable water. Efficient encapsulation is due to rapid diffusion of $\mathrm{AgNO}_{3}$ into the swollen hydrogel owing to their small size and poor release of $\mathrm{AgNO}_{3}$ from the hydrogel is attributed to strong chelation of silver ion with the $-\mathrm{OH}$ and $-\mathrm{NH}_{2}$ of chitosan.

Mafenide acetate (MFA) encapsulation and release. As seen in Figure 5 and 6 encapsulation and release of MFA was $\mathrm{pH}$ dependent and followed the $\mathrm{pH}$ dependent swelling trend. Release was monitored using those hydrogels that encapsulated $26-28 \mathrm{mg}$ of mafenide acetate. Encapsulation and release was most efficient for the Quat-188 modified chitosan at $\mathrm{pH} 5.5$ (DI water), making it an ideal candidate to serve as controlled release vehicle for skin. Efficiency of encapsulation and release increased with the increase in the amount of freezable water and the extent of swelling. However, the rate of MFA incorporation was slower that $\mathrm{AgNO}_{3}$ incorporation though the semi-IPNs swelled to the same extent for both these encapsulates. Smaller size and affinity of silver ion to chelate with chitosan might have aided $\mathrm{AgNO}_{3}$ to diffuse faster into the hydrogel. It should also be noted that though the modified and the unmodified hydrogels were swollen more than $100 \%$, complete release of mafenide acetate was not observed, which is probably due to the formation of hydrogen bonding between MFA and the hydrogel. Thus it can be stated that apart from swelling potential of the hydrogel, the interaction

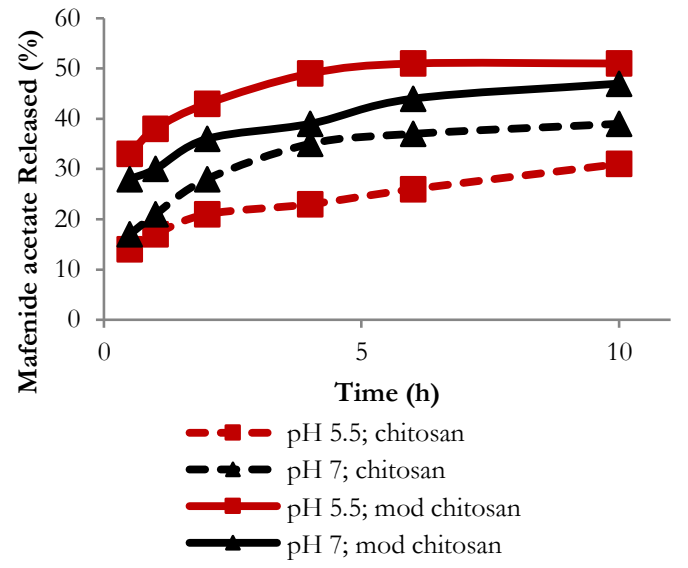

Figure 6. Mafenide acetate release from chitosan and modified chitosan PAA semi IPNs as function of time and $p H$.

between the drug and the hydrogel and the amount of freezable water in the hydrogel also have profound influence on encapsulation and release behavior.

\section{CONCLUSION}

Semi IPN network of chitosan and PAA was synthesized in one step. Strength of the semiIPN hydrogels increased with the increase in the amount of chitosan and upon modification of chitosan with Quat-188. Modification improved the $\mathrm{pH}$ dependent swelling behavior and showed better encapsulation and release potential. Encapsulation of $\mathrm{AgNO}_{3}$ was much faster than mafenide acetate, however hardly any release of $\mathrm{AgNO}_{3}$ was observed, whereas mafenide acetate got release at a controlled rate, proving that synthesized semi-IPN can be explored further as patch for encapsulation and release of mafenide acetate alone for topical application. Strong interaction between the drug and the matrix will facilitate efficient encapsulation but might adversely affect the release rate. Thus chitosan PAA semi-IPN hydrogel can serve as an efficient vehicle for the encapsulation and release of encapsulates such as MFA however it cannot be used as controlled release vehicle for drugs such as $\mathrm{AgNO}_{3}$ which has strong affinity for the encapsulating vehicle.

This study has established that it is essential to consider drug-polymer interaction while 
formulating a controlled release device. Encapsulation and release potential cannot be gauged from swelling profile alone.

The study reported is preliminary in nature. More experiments are required to be performed to establish the potential of chitosan poly(acrylic acid) semi-IPN serve as a wound dressing patch for topical delivery of the drug.

\section{ACKNOWLEDGMENT}

The authors would like to acknowledge the Loyola School faculty grant for scholarly work committee for financial support and Ateneo LS Bookstore Professorial Chair.

\section{REFERENCES}

Ahn J-S, Choi HK, Chun M-K, Ryu J-M, Jung $\mathrm{J}-\mathrm{H}, \mathrm{Kim}$ Y-U. Cho, C-S. Release of triamcinolone acetonide from mucoadhesive polymer composed of chitosan and poly(acrylic acid) in vitro. Biomaterials, 2002; 23(6): 1411-1416.

Bochicchio G, Kilbourne M, Kuehn R, Keledjian K, Hess J, Scalea T. Use of a modified chitosan dressing in a hypothermic Coagulopathic grade V liver injury model. Am J Surg. 2009; 198(5), 617-622.

Dash M, Ferri M, Chiellini F. Synthesis and characterization of semi-interpenetrating polymer network hydrogel based on chitosan and poly(methacryloylglycylglycine). Mater Chem Phys. 2012; 135, 1070-1076.

Dragan ES, Perju MM, Dinu MV. Preparation and characterization of IPN composite hydrogels based on polyacrylamide and chitosan and their interaction with ionic dyes. Carbohyd Polym. 2013; 88(1), 270-281.

Guo B-L, Gao Q-Y. Preparation and properties of a $\mathrm{pH} /$ temperature-responsive carboxymethyl chitosan/poly(N-isopropyl acrylamide)semi-IPN hydrogel for oral delivery of drugs. Carbohyd Res. 2007; 342(16), 2416-2422.
Henares TG, Cuyegkeng MAC. Controlled Release ot Methvl Salicvlate in ChitosanPolv(N-Isopropvlacrvlamide) SemiInterpenetrating Networks. Kimika, 2010, 23(1), 26-31.

$\mathrm{Hu}$ C, Li B, Guo R, Wu H, Jiang Z. Pervaporation performance of chitosanpoly(acrylic acid) polyelectrolyte complex membranes for dehydration of ethylene glycol aqueous solution. Sep Purif Technol 2007; 55(3), 327-334.

Jayakumar R, Ramachandran R, Divyarani VV, Chennazhi KP, Tamura H. Nair SV. Fabrication of chitin-chitosan/nano $\mathrm{TiO}_{2-}$ composite scaffolds for tissue engineering applications. Int J Biol Macromol. 2011;48(2), 336-344.

Jia Z, Wu, J. A thermo- and $\mathrm{pH}$-sensitive hydrogel composed of quaternized chitosan/ glycerophosphate. Int J Pharm. 2006; 315, 1-11.

Li P, Wang Y, Peng Z, She F, Kong, L. Development of chitosan nanoparticles as drug delivery systems for 5-fluorouracil and leucovorin blends. Carbohydr Polym. 2011; 85(3), 698-704.

Li Y, Wang Y, Wu D, Zhang K. Hu, Q. A facile approach to construct three-dimensional oriented chitosan scaffolds with in-situ precipitation method. Carbohydr Polym. 2010; 80(2), 408-412.

Njagi J, Erlichman JS, Aston JW, Leiter JC, Andreescu S. A sensitive electrochemical sensor based on chitosan and electropolymerized Meldola blue for monitoring NO in brain slices. Sensors Actuat B: Chem. 2010; 143(2), 673-680.

Odaci D, Timu S, Telefoncu A. Bacterial sensors based on chitosan matrices. Sensors and Actuat B: Chem. 2008; 134(1), 89-94.

Park JH, Saravanakumar G, Kim K, Kwon IC. Targeted delivery of low moleculardrugs using chitosan and its derivatives. Adv Drug Deliver Rev. 2010; 62(1), 28-41. 
Qin C, Xiao Q, Li H, Fang M, Liu Y, Chen X, $\mathrm{Li}$ Q. Calorimetric studies of the action of chitosan-N-2-hydroxypropyl trimethyl ammonium chloride on the growth of microorganisms. Int Journ Bio Macro. 2004; 34,121-126.

Quiñones JP, Szopko R, Schmidt C, Covas CP. Novel drug delivery systems: Chitosan conjugates covalently attached to steroids with potential anticancer and agrochemical activity. Carbohydr Polym. 2011; (3),858-864.

Sailaja GS, Ramesh P, Kumary TV, Varma HK. Human osteosarcoma cell adhesion behaviour on hydroxyapatite integrated chitosan-poly(acrylic acid) polyelectrolyte complex. Acta Biomater. 2006; 2, 651-657.

Sajomsang W, Tatayanona S, Tangpasuthadol V, Daly WH. Quaternization of N-aryl chitosan derivatives: synthesis, characterization. Carbohydr Res. 2009; 344, 2502-2511
Nakaoki T, Yamashita H. Bound States of Water in Poly(vinyl alcohol) Hydrogel prepared by Repeated Freezing and Melting Method. J Mol Struct. 2008; 875,282-287.

Nakano T, Nakaoki T. Coagulation Size of freezable Water in Poly(vinyl alcohol) Hydrogels formed by different Freeze/thaw Cycle Periods. Polym J. 2011; 43,875-880.

Wu HD, Yang JC, Tsai T, Ji DY, Chang WJ, Chen CC, Lee SY. Development of a chitosan-polyglutamate based injectable polyelectrolyte complex scaffold. Carbohydr Polym. 2011; 85(2), 318-324. 